\title{
Nanoscale control of interfacial processes for latent fingerprint enhancement
}

\author{
Rachel M. Sapstead (nee Brown), ${ }^{a}$ Karl S. Ryder, ${ }^{a}$ Claire Fullarton, ${ }^{a}$ \\ Maximilian Skoda, ${ }^{b}$ Robert M. Dalgliesh, ${ }^{b}$ Erik B. Watkins, ${ }^{c}$ \\ Charlotte Beebee, ${ }^{c}$ Robert Barker, ${ }^{c}$ Andrew Glidle ${ }^{d}$ \\ and A. Robert Hillman*a
}

Received 15th April 2013, Accepted 17th May 2013

DOI: $10.1039 / c 3 f d 00053 b$

Latent fingerprints on metal surfaces may be visualized by exploiting the insulating characteristics of the fingerprint deposit as a "mask" to direct electrodeposition of an electroactive polymer to the bare metal between the fingerprint ridges. This approach is complementary to most latent fingerprint enhancement methods, which involve physical or chemical interaction with the fingerprint residue. It has the advantages of sensitivity (a nanoscale residue can block electron transfer) and, using a suitable polymer, optimization of visual contrast. This study extends the concept in two significant respects. First, it explores the feasibility of combining observation based on optical absorption with observation based on fluorescence. Second, it extends the methodology to materials (here, polypyrrole) that may undergo postdeposition substitution chemistry, here binding of a fluorophore whose size and geometry preclude direct polymerization of the functionalised monomer. The scenario involves a lateral spatial image (the whole fingerprint, first level detail) at the centimetre scale, with identification features (minutiae, second level detail) at the 100-200 $\mu \mathrm{m}$ scale and finer features (third level detail) at the 10-50 $\mu \mathrm{m}$ scale. However, the strategy used requires vertical spatial control of the (electro)chemistry at the 10-100 $\mathrm{nm}$ scale. We show that this can be accomplished by polymerization of pyrrole functionalised with a good leaving group, ester-bound FMOC, which can be hydrolysed and eluted from the deposited polymer to generate solvent "voids". Overall the "void" volume and the resulting effect on polymer dynamics facilitate entry and amide bonding of Dylight 649 NHS ester, a large fluorophore. FTIR spectra demonstrate the spatially integrated compositional changes. Both the hydrolysis and fluorophore functionalization were followed using neutron reflectivity to determine vertical spatial composition variations, which control image development in the lateral direction.

\footnotetext{
${ }^{a}$ University of Leicester, LeicesterLE1 7RH. E-mail: arh7@le.ac.uk ${ }^{b}$ Rutherford Appleton Laboratory, Didcot, Oxfordshire, OX11 OQX, UK 'Institut Laue Langevin, Grenoble, Cedex 9, France ${ }^{d}$ Glasgow University, Glasgow, G12 8LT, UK
} 


\section{Introduction}

Fingerprints provide an example of pattern formation in nature, carrying information that uniquely identifies an individual. Notwithstanding the rise of sophisticated genetic methods, they remain the cornerstone of many criminal investigations and have a number of non-criminal applications based upon identification of an individual. ${ }^{1}$ The efficacy of this approach is in large measure associated with the complexity of a fingerprint and the consequent practical difficulty of forgery; powerful software tools for analysis and recognition facilitate exploitation of this potential. The power of fingerprint evidence for analytical purposes in general is anecdotally recognized by the use of the term in other contexts, such as the "fingerprint" region of IR spectra and DNA "fingerprinting". The challenge in realizing this analytical opportunity lies in visualization of the interfacial chemical transfer that constitutes a fingerprint, primarily in the case of latent fingerprints, for which the nature ${ }^{2}$ and extent $^{3}$ of the deposit mean that they are not immediately visible to the eye. Here we explore how this can be accomplished by a novel electroanalytical approach based upon spatially selective deposition of electroactive polymers with variable optical properties. The intellectual novelty lies in the need to control the (electro)chemistry on different length scales and in both the lateral and vertical directions.

When a finger contacts a surface, exchange of material with the surface leaves behind a trace of this contact which resembles the pattern present on the finger. Dependent on the substrate and the nature of the contact, the fingerprint may be visible, latent or plastic. ${ }^{4}$ Since they are not immediately visible to the eye, and thus less readily "wiped", latent fingerprints are the greatest source of forensic evidence. To give an indication of scale, in the UK on the order of 700000 objects are fingerprinted per annum. In response to this demand, numerous methods and reagents have been developed to effect latent fingerprint visualization but, perhaps surprisingly, the operational success rate is only $c a .10 \%$. While there may be local variations in demand and/or success rate for specific types of object, the global need for improvement is clear.

The existence of fingerprint patterns was recognized in ancient times: ${ }^{5,6}$ they have been identified on hand-formed building materials in Jericho dating back to $7000 \mathrm{BC}$ and on the reverse of Chinese clay seals from 300 BC. Much later they were used as accompaniments to signatures by citizens claiming damages following the siege of Londonderry in $1691^{5}$ and by the engraver Bewick who used an engraved fingerprint as a signature on his work. ${ }^{6}$ The first documented study of fingerprints was in the 17 th century by Grew, ${ }^{5}$ followed by attempts at pattern classification in 1823 by Purkinje, ${ }^{5,6}$ and later by Henry. ${ }^{5}$

Development of these historical observations for forensic application was reliant upon three crucial deductions made during the 19th century. First, Herschel $^{7}$ made the critical observation - on his own hands - that fingerprints do not change during the life of an individual. ${ }^{5}$ Indeed, the friction ridge skin pattern that constitutes a fingerprint persists after death, thereby enabling post mortem identification. ${ }^{8}$ Second, by removing skin from the fingers and allowing it to re-grow, it was demonstrated that injury does not change fingerprint patterns. ${ }^{5}$ Third, in 1892 Galton $^{9}$ estimated that the odds of two individuals having identical fingerprints were 64 billion to 1 ; thus, for all practical purposes, they are unique to an individual. Combination of these observations pointed to the value of 
fingerprints in criminal investigations. ${ }^{10}$ The outcome of this, in the first decade of the 20th century, was establishment of the UK's first fingerprint bureau ${ }^{5,7}$ and the use of fingerprint evidence to secure a murder conviction. ${ }^{6}$

Fingerprint patterns fall into three basic categories (so-called first level detail): loops, whorls and arches., ${ }^{5,11}$ While differences at this gross level can clearly eliminate certain individuals, positive identification relies on the minutiae (or second level detail) within the pattern: these include such features as ridge endings, crossovers (bridges), short independent ridges, islands, bifurcations, spurs, dots and lakes. ${ }^{5}$ The standard for matching a crime scene fingermark to one from a database varies with jurisdiction: in some cases there is a set minimum number of points of similarity (although the number is not universal) and in others (including the UK) it is decreed a matter for a recognized expert to decide. As a rule of thumb, identification of around 16 points of similarity can be expected to be considered conclusive. ${ }^{712}$ There are also finer features (third level detail) present in a fingerprint image: these include the detailed shapes of the ridges and individual sweat pores. While not currently used in fingerprint identification, there is considerable research interest in third level detail, since it may in future permit analysis of smaller fragments of marks left by a finger, i.e. partial fingerprints.

Here we focus on latent fingerprints, since these are the primary source of forensic evidence. In general terms, the traditional approach has been to apply a reagent that interacted with the residues left by contact of the finger. We do not rehearse the diverse methods available, since these have been reviewed elsewhere recently, ${ }^{13-15}$ but rather show how the apparently diverse methods used in fact have some similarities that limit their efficacy and motivate the novel approach developed here. The classical approach is to apply a powder (either dry or as a suspension) that adheres physically to the sweat residues; the powder may be fluorescent, ${ }^{16-18}$ magnetic ${ }^{19,20}$ or thermoplastic. In each case, this method is reliant upon there being sufficient residue present for adhesion of a powder with adequate optical contrast to the substrate. ${ }^{21-26}$ More recent developments involve (by dipping, spraying or gas phase delivery, according to the chemistry) ninhydrin solution, ${ }^{27}$ vacuum metal deposition, ${ }^{21}$ small particle reagent,${ }^{28}$ physical developer ${ }^{28}$ cyanoacrylate ("superglue") polymerization in conjunction with a suitable dye, ${ }^{29,30} \mathrm{~S}_{2} \mathrm{~N}_{2}$ polymerization ${ }^{31}$ and cadmium sulphide nanocomposites. ${ }^{32}$ In some cases the interaction of the reagent may be relatively unspecific (physical adhesion of powders), in others it may involve moderately specific chemistry (CdS binding with fatty acids and amino acids generally found in fingerprint deposits) and in other cases it may be very specific (reaction with secreted drug metabolites of antibody-functionalised nanoparticles. ${ }^{33}$ However, the common factor is that the reagent interacts with the deposited residue. This makes all these technologies vulnerable to loss or deterioration of fingerprint residue, e.g. as a consequence of ageing, environmental exposure or abuse (attempted washing).

The approach developed here is complementary to those described above, in that the "reagent" is applied to the bare substrate surface that lies between the deposited fingermark ridges. The means of accomplishing this is to use the fingerprint deposit as a "mask" or template, whose broadly insulating characteristics preclude electron transfer from a metal substrate to a solution precursor. Since electron tunnelling can only take place over very short distances, only a very thin layer (ca. 1-2 nm) of fingerprint residue is required - far less (by an order of magnitude) than required in the conventional strategies listed above. We have recently demonstrated 
proof-of-concept for this strategy ${ }^{34,35}$ in the context of electropolymerization of aromatic solution precursors ${ }^{36}$ to generate conducting polymer films. Other applications of electrochemically-based methods of latent fingerprint visualization include imaging using the SECM ${ }^{38-41}$ and deposition of Au nanoparticles. ${ }^{42}$

In the case of electroactive polymer enhancement, after transfer to a background electrolyte, the electrochromic properties of these materials were exploited to adjust the visual contrast between the polymer and the metal substrate. Evaluation of the ability of this approach to visualize fingerprints subject to ageing in a range of environments suggests that there are practically relevant situations in which the methodology may be superior to currently employed methods, for example involving powders and cyanoacrylate. ${ }^{37}$

The aim of the present study is to extend the concept of the electrochromic polymer enhancement strategy in two significant respects. First, we wish to explore the possibility of combining observation based on optical absorption (as above) with observation based on emission. Since the advantages, notably sensitivity, of fluorescence detection are appreciated in fingerprint visualization, translation to practical application would be facilitated by existing instrumentation. In the context of "superglue" enhancement, subsequent use of fluorescent dyes is a necessity, since the cyanoacrylate polymer is not coloured. This leads naturally to our second generic goal, use of electropolymerized films permitting excellent spatial control but with sub-optimal optical properties. In particular, we wish to move from thiophene ${ }^{35}$ and aniline ${ }^{34}$ based materials to pyrrole-based systems, for which there is much greater opportunity for manipulation of properties by substituent chemistry on the $-\mathrm{N}-\mathrm{H}$ functionality. Here we focus on the underlying fundamental (electro)chemistry of this approach.

The future viability of enhancement and analysis of latent fingerprints using this strategy relies on "writing" (electropolymerization and post-deposition reaction) and "reading" (absorption and emission observations) of spatial information at different length scales. The precise figures will vary from one individual to another, but typically this is at $c a .1 \mathrm{~cm}$ for first level detail (the whole print), at 100-200 $\mu \mathrm{m}$ for second level detail and at 10-50 $\mu \mathrm{m}$ (third level detail). These feature sizes set the chemical challenge: control of enhancement chemistries with commensurate lateral spatial resolution to the feature size. However, the requirement for vertical resolution is somewhat different. Typical fingerprint deposits may be a few microns thick as-deposited, but evaporative and other environmental losses will typically decrease this to $100 \mathrm{~s}$ of $\mathrm{nm}$ before enhancement is undertaken. This defines the vertical resolution required for the (electro) chemistry: there is an optimum to be found between the lower limit of detection of deposited material and over-filling ${ }^{35}$ of the trenches between fingerprint ridge deposits. To summarize, this singular analytical challenge of uniquely identifying an individual based on electroanalytical visualization of their fingerprint requires simultaneous control of (electro)chemical processes on length scales from the nanometre to the centimetre regime and in both lateral and vertical directions.

\section{Experimental}

\section{Reagents, materials and electrodes}

$\mathrm{N}$-Cyanoethylpyrrole (PyCN), piperidine, tetrabutylammonium perchlorate (TBAP), perchloric acid, hydrogenous and deuterated acetonitrile were all used as supplied 
(Sigma Aldrich). N-Aminopropylpyrrole $\left(\mathrm{PyNH}_{2}\right)$ was synthesised via reduction of PyCN $(0.056 \mathrm{~mol})$ with $\mathrm{LiAlH}_{4}(0.26 \mathrm{~mol})$ in anhydrous ether. The reaction was stirred for $2 \mathrm{~h}$ before excess $\mathrm{LiAlH}_{4}$ was neutralised with water. The solution was dried over $\mathrm{MgSO}_{4}$ before evaporating to dryness to yield a yellow oil (84\% yield).

(9H-Fluoren-9-yl)methyl-3-(1H-pyrrol-1-yl)propylcarbamate (PyFMOC) was made using a Merrifield synthesis. FMOC-Cl $(0.021 \mathrm{~mol})$ in water-dioxane $(60 \mathrm{ml}, 1: 1)$ was added to a solution of $\mathrm{PyNH}_{2}(0.021 \mathrm{~mol})$ and $\mathrm{Na}_{2} \mathrm{CO}_{3}(0.028 \mathrm{~mol})$ in waterdioxane $(60 \mathrm{ml}, 1: 1)$ in an ice bath. The solution was then stirred at room temperature for $2 \mathrm{~h}$. PPyFMOC was extracted with ethyl acetate and dried over $\mathrm{MgSO}_{4}$ before evaporating to dryness to yield a white solid (78\% yield).

For the neutron reflectivity (NR) experiments, the working electrode was prepared by sputter coating gold onto a polished single-crystal quartz block $(100 \times 50 \mathrm{~mm}$, Gooch and Housego $)$ coated with a monolayer of 3-mercaptopropyltrimethoxysilane (MPTS) (Sigma Aldrich) to promote adhesion. The nominal Au film thickness was $20 \mathrm{~nm}$. For other experiments, the electrodes were metal sheet, as indicated in the figure legends. The counter electrode was in each case a Pt gauze, of adequate size to ensure that the counter electrode reaction was not limiting. The reference electrode was a double junction $\mathrm{Ag}|\mathrm{AgCl}| \mathrm{KCl}$ (saturated) electrode. These were assembled into a standard three electrode cell configuration; for the NR measurements, the purpose built cell has been described elsewhere. ${ }^{43,44}$

\section{Instrumentation}

NR measurements were performed on FIGARO ${ }^{45}$ and D17 at the Institut LaueLangevin (Grenoble, France) and on INTER ${ }^{46}$ at the ISIS Facility of the Rutherford Appleton Laboratory (Harwell Oxford, UK). Static neutron reflectivity measurements were performed ex situ in air ("dry") and in situ immersed in $\mathrm{h}_{3^{-}}$and $d_{3}$-acetonitrile before and after the film fabrication stage (see Fig. 3, below). Deuterated solvents were used to maximise contrast between the polymer and the electrolyte so that the solvation within the polymer could be probed. Kinetic measurements were recorded during the hydrolysis stage of the reaction (see Fig. 3), using time-of-flight instrumentation with $\lambda$ ranges of $2-30 \AA$ (ILL) and 1.5-16 $\AA$ (ISIS). Using different incident angles, this provides an accessible momentum transfer range of $0.004<Q / \AA^{-1}<0.12$, where $Q$ is defined as $(4 \pi / \lambda)$ $\sin \theta ; \lambda$ is the wavelength of the neutron and $\theta$ is the incident angle. (Strictly, this is $Q_{z}$ but since we only consider specular reflection we use the simpler notation $Q$.) The collimation slits were set to give a beam footprint on the sample of $60 \mathrm{~mm} \times$ $30 \mathrm{~mm}$; they also define the $\Delta \theta / \theta$ resolution. The $\Delta \lambda / \lambda$ resolution is dependent on the source (spallation source at ISIS, reactor at ILL) and associated instrumentation (chopper settings at ILL). In both cases, the resultant resolution in momentum transfer was $\Delta Q / Q \sim 2-3 \%$. Data acquisition times were $c a .1 .5 \mathrm{~h}$ per static run and $10 \mathrm{~min}$ for kinetic runs.

Photographs were taken with a Canon A480 digital camera and were digitally enhanced using the GNU Image Manipulation Program 2.6.7. (G.I.M.P.). 3D profiles were recorded with a Zeta 200 Optical Profiler. Reflectance FTIR spectra were acquired with p-polarised radiation incident at a reflectance angle of $55^{\circ}$ using a Spectra-Tech reflectance accessory mounted on a Bomem MB120 infra-red instrument. 


\section{Procedures}

Poly(3,4-ethylenedioxythiophene) (PEDOT) films were deposited potentiostatically $(E=0.90 \mathrm{~V})$ from an aqueous solution containing $0.01 \mathrm{M}$ EDOT monomer in $0.01 \mathrm{M}$ SDS-0.1 $\mathrm{M} \mathrm{H}_{2} \mathrm{SO}_{4}$. Polypyrrole (PPy) films were deposited potentiostatically $(E=0.90 \mathrm{~V})$ from an aqueous solution containing $0.01 \mathrm{M}$ pyrrole monomer in $0.1 \mathrm{M} \mathrm{LiClO}_{4}$. In both cases, the film thickness was controlled by deposition time. PPyFMOC and $\mathrm{PPyNH}_{2}$ films were deposited, respectively, from $10 \mathrm{mM}$ PyFMOC in $0.1 \mathrm{M}$ TBAP- $\mathrm{CH}_{3} \mathrm{CN}$ and $10 \mathrm{mM} \mathrm{PyNH}_{2}$ in $0.1 \mathrm{M}$ TBAP- $\mathrm{CH}_{3} \mathrm{CN} / \mathrm{HClO}_{4}(\mathrm{pH} 4.5)$. The films were grown potentiodynamically $\left(\nu=20 \mathrm{mV} \mathrm{s}^{-1}\right)$ in the potential range $0.3<E / \mathrm{V}<E_{\max }$, where the anodic limit $E_{\max }$ (typically $1.15 \mathrm{~V}$ ) was set to cap the anodic current to $4 \mathrm{~mA}$. This procedure was designed to avoid uncontrollably rapid growth; the amount of deposited polymer was varied with adequately fine control by varying the number of deposition cycles. All measurements were made at room temperature, $20 \pm 2{ }^{\circ} \mathrm{C}$. PPyFMOC films were deprotected using a $30 \% \mathrm{v} / \mathrm{v}$ piperidine solution in $\mathrm{CH}_{3} \mathrm{CN}$ to yield $\mathrm{PPyNH}_{2}$. $\mathrm{PPyNH}_{2}$ films were reacted with 0.01 M Dylight 649 NHS ester (hereafter referred to as "Dylight", for brevity) (Thermo Scientific) in DMSO-pH7 phosphate buffer in water $(1: 9 \mathrm{ml})$.

\section{Data analysis}

The principles of neutron reflectivity data analysis ${ }^{47}$ and the issues arising for samples involving "wet" interfaces under electrochemical control ${ }^{48}$ have been described elsewhere. The variation with momentum transfer of reflectivity from an interface, $R(Q)$, is determined by the depth profile of the scattering length density, $N b$, where $N$ represents the concentration of scattering atoms present and $b$ is their scattering length; the value of $b$ is isotopically unique and medium independent. The scattering length density of a composite medium, here a solvated polymer film, is a weighted sum of the $N b$ values of its components: film composition determines scattering length density and thence reflectivity. Experimentally, we invert the process and use reflectivity, $R$, to determine composition - in practice, the volume fractions of polymer and solvent - by model fitting the reflectivity profile, $R(Q)$. This was accomplished using the box-model approach, implemented in the Motofit software. ${ }^{49}$

\section{Results}

\section{Electrochromic enhancement of latent fingerprints - basic observations in absorption mode}

Before attempting to exploit the substitution chemistry opportunities presented by the $>\mathrm{N}-\mathrm{H}$ function in pyrrole, it is first necessary to demonstrate that PPy films can in fact be deposited with spatial selectivity directed by a fingermark on a metallic substrate. Fig. 1 and Fig. 2 show representative images of two fingermarks on 304 stainless steel substrates, following enhancement by electrodeposition of PEDOT and PPy, respectively. In panel (a) of each figure, the paler (nominally white) regions correspond to the fingermark itself, i.e. the complex mixture of materials secreted from pores along the ridges on the fingertip. The darker regions (blue for PEDOT in Fig. 1 and black for polypyrrole in Fig. 2) correspond to polymer deposited on the bare metal between the fingerprint deposits. The PEDOT enhanced image acts as a control; this material has 


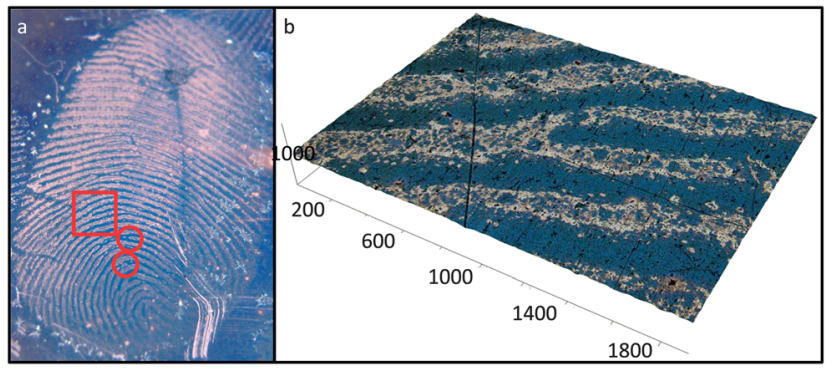

Fig. 1 PEDOT enhanced sebaceous fingerprint on 304 stainless steel. Deposition conditions as in main text; deposition time, $t_{\text {dep }}=3000 \mathrm{~s}$. Panel (a): whole fingermark image. Dark (blue) regions correspond to PEDOT and lighter regions to fingerprint deposit. Circles highlight examples of second level detail, a bifurcation and a ridge ending. Panel (b): optical microscope image of selected area from panel (a) (as defined by the rectangle). Larger dark circular regions within light areas represent individual sweat pores. Vertical and horizontal distance scales (expressed in $\mu \mathrm{m}$ ) are relative.

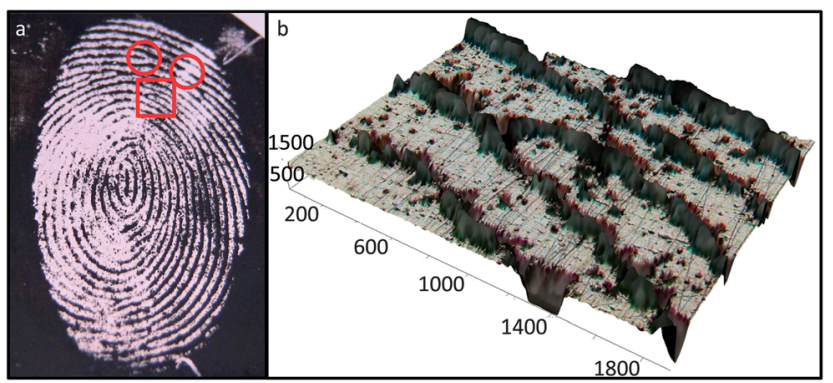

Fig. 2 PPy enhanced sebaceous fingerprint on 304 stainless steel. Deposition conditions as in main text; deposition time, $t_{\text {dep }}=3000 \mathrm{~s}$. Panel (a): whole fingermark image. Dark regions correspond to PEDOT and lighter regions to fingerprint deposit. Circles highlight examples of second level detail, a crossover and a ridge ending. Panel (b): optical microscope image of selected area from panel (a) (as defined by the rectangle). Larger dark circular regions within light areas represent individual sweat pores. Vertical and horizontal distance scales (expressed in $\mu \mathrm{m}$ ) are relative.

previously been demonstrated to provide good visualization of latent fingermarks, with high fidelity and controllable visual contrast. ${ }^{35}$

We have quite deliberately shown examples that might be typical of real evidence, rather than highly controlled ("groomed") model examples. Thus, one can see evidence of damage to the fingermark and of adjacent fingermarks in Fig. 1 and of smearing (for example, caused by motion of the finger on the surface) and variable amounts of residue in Fig. 2. These and other imperfections represent practical challenges to be addressed.

At the coarsest level of interpretation, first level detail, the fingerprints in Fig. 1 and Fig. 2 are, respectively, a loop and a whorl. While this is clearly not sufficient for identification purposes, it is clear that there is much second level detail present within these images. Panel (b) in each of Fig. 1 and Fig. 2 shows higher magnification optical images of the samples in panels (a). By any reasonable standard (see above), it would be possible to achieve an evidentially acceptable identification from images such as these using various combinations of second level detail. 
To illustrate the principle, we simply identify two such features on each image. It is also possible to identify a large number of pores, seen as dots along the ridges where the absence of contact with the substrate permits polymer deposition; these are examples of third level detail.

\section{Overview of extension to emission mode visual enhancement}

The qualitative conclusion of the preliminary experiments shown above is that polypyrrole can be added to the set of electrochromic materials (to date, polyaniline ${ }^{34}$ and PEDOT $^{35}$ ) that permit visualization of latent fingermarks on metallic substrates by means of their optical absorption properties; note that the work of Bersellini ${ }^{36}$ in this respect demonstrated the facility to electrodeposit polypyrrole, but did not go on to exploit its electrochromic properties. We therefore proceed to the more challenging goal of developing a polymeric system with suitable absorption and fluorescence characteristics. Conceptually, the simplest approach is to functionalise the nitrogen of the pyrrole ring with a fluorophore. In practice, the (necessarily) large size of most fluorophores creates such steric hindrance that the substituted pyrrole monomers cannot polymerize. We therefore arrive at the three step strategy schematically represented in Fig. 3.

The essential idea is to functionalize pyrrole units with the fluorophore postdeposition. However, in order to accomplish this, there is still a requirement to create sufficient free volume within the polymer film to accommodate the fluorophore units. The tactic employed is to polymerize not pyrrole itself, but an $\mathrm{N}$-functionalized derivative that can readily be removed post-deposition. The balance to be struck is use of a substituent that is not so large as to preclude polymerization but that is large enough to create appreciable free volume. The functionality chosen was the widely used FMOC protecting group. Thus, we set out to polymerize the $N$-substituted FMOC derivative of pyrrole (PyFMOC), then hydrolyze and leach out the protecting group to leave an amine-functionalized polypyrrole film. The semi-fluid nature of the polymer film means that the
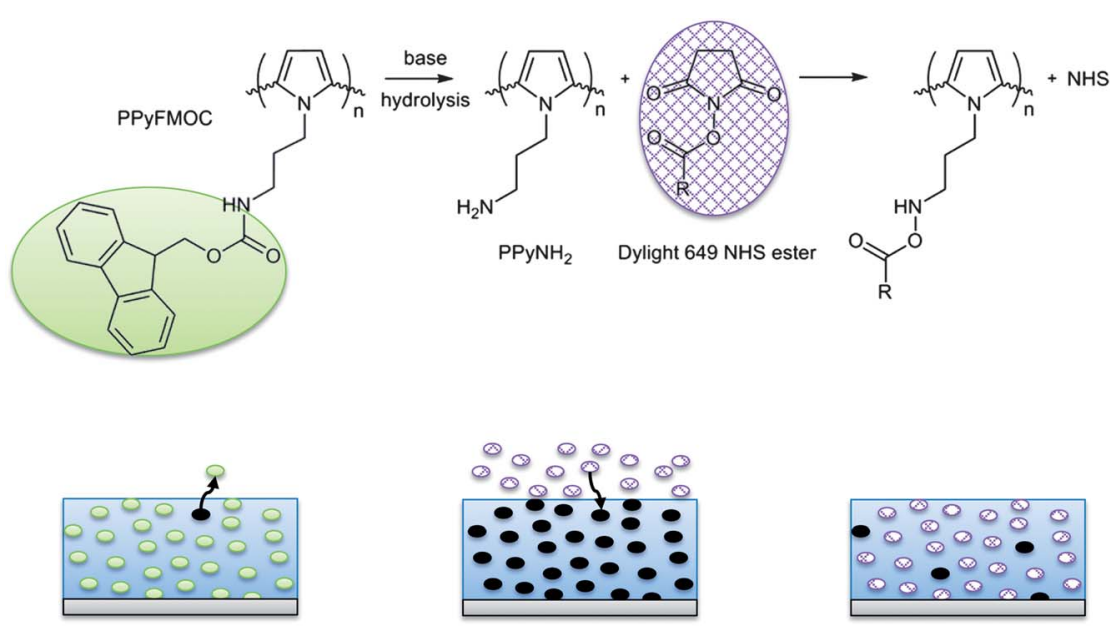

Fig. 3 Cartoon representation of (i) PPy-FMOC deposition, (ii) FMOC hydrolysis to create solvent "voids"; (iii) diffusion of fluorophore into voids and attachment to polymer. Stylised ellipsoids (simplistically, of similar size) represent FMOC (pale green), solvent (black) and fluorophore (diamond chequered). 
randomly distributed free volume can aggregate to generate "voids" of sufficient size to accommodate larger fluorophore moieties. Choice of a fluorophore with an ester functionality provides the means of covalent bonding by amide formation (see Fig. 3).

In the experimental realisation of the strategy indicated in Fig. 3, critical issues are the completion of hydrolysis, the extent to which departing FMOC is replaced by solvent $c f$. film contraction, the vertical (perpendicular to the interface) spatial distribution of replaceable solvent and the penetration of fluorophore into the film. The outcomes, which will ultimately determine performance, are addressed using a combination of spectroscopic and neutron reflectivity measurements.

\section{Optimization of film deposition}

The limited aim of this study is establishment and assessment of the strategy of Fig. 3 on clean (i.e. non-fingermarked) metal surfaces. The deliberate absence of lateral spatial variation focuses attention on the required control of film composition in the vertical direction. Fig. 4 and Fig. 5, respectively, show the voltammetric responses of $\mathrm{PPyFMOC}$ and $\mathrm{PPyNH}_{2}$ films on $\mathrm{Au}$ during electropolymerization (panels (a)) and after transfer to background electrolyte (panels (b)). Both sets of responses provide information relating to polymer deposition. In the former instance, the integrated current provides cycle-by-cycle monitoring to facilitate deposition of the chosen amount of polymer. In practice, use of the cathodic half cycle response is better, since this is not complicated by contributions from the (irreversible) anodic polymerization current contribution.

The coulometric assay described above is reliant upon complete film redox conversion on the experimental timescale; that this is accomplished is demonstrated by the data in Fig. $4 \mathrm{~b}$ and Fig. 5b. In these measurements (following completion of film deposition) there is no monomer present in the solution, so the issue of distinguishing polymerization and film redox chemistry is irrelevant. For films of suitable coverage, i.e. appropriate both to the NR experiment (giving multiple well-defined interference fringes within the accessible $Q$ range) and to future forensic exploitation (not so thick as to obscure all image detail when a fingermark is present), we find that the peak currents are linearly proportional to the potential scan rate (see Fig. 4c and Fig. 5c). This indicates complete redox
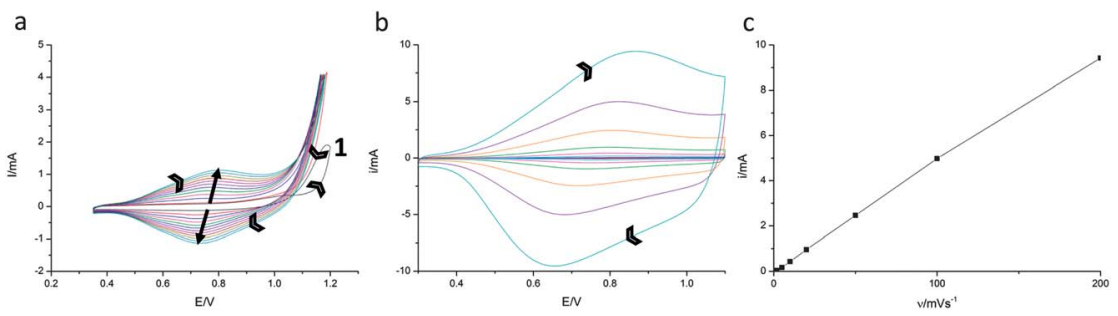

Fig. $4 \quad i-E$ responses for a PPyFMOC film. Panel (a): during potentiodynamic electropolymerization $(v=$ $20 \mathrm{mV} \mathrm{s}^{-1}$ ); panel (b): after transfer to a monomer-free background electrolyte (subsequent to the final cycle of panel (a)), during cycling at $v=1,2,5,10,20,50,100$ and $200 \mathrm{mV} \mathrm{s}^{-1}$ (increasing "outwards"); panel (c): variation of cathodic peak current (from curves in panel (b)) with scan rate. Solution compositions as described in the main text. In panels (a) and (b), chevron arrows indicate scan direction. In panel (a) large arrows indicate the time sequence and "1" indicates the first deposition cycle (note the nucleation loop). 

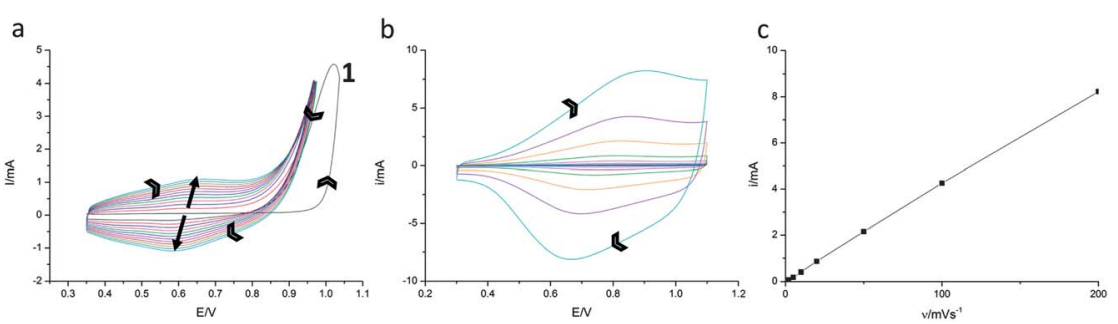

Fig. $5 i-E$ responses for a $\mathrm{PPyNH}_{2}$ film. Panel (a): during potentiodynamic electropolymerization $\left(v=20 \mathrm{mV} \mathrm{s}^{-1}\right)$; panel (b): after transfer to a monomer-free background electrolyte (subsequent to the final cycle of panel (a)), during cycling at $v=1,2,5,10,20,50,100$ and $200 \mathrm{mV} \mathrm{s}^{-1}$ (increasing "outwards"); panel (c): variation of cathodic peak current (from curves in panel (b)) with scan rate. Solution compositions as described in main text. In panels (a) and (b), chevron arrows indicate scan direction. In panel (a) large arrows indicate the time sequence and "1" indicates the first deposition cycle (note the nucleation loop).

conversion of the film on the experimental timescale, validating a coulometric assay of the spatially integrated surface population of polymer. On this basis, the surface coverage, $\Gamma / \mathrm{mol} \mathrm{cm}^{-2}$, is determined as $q / n F A$, where $q / C$ is the charge, $n$ is the number of electrons transferred ("doping level", $n=0.33)^{50}, A$ is the electrode area and $F$ is the Faraday constant. For the data shown, the final polymer coverages are $\Gamma_{\text {PPyलMOC }}=20 \mathrm{nmol} \mathrm{cm}{ }^{-2}$ and $\Gamma_{\text {PPyNH2 }}=19 \mathrm{nmol} \mathrm{cm}^{-2}$, where in both cases the surface population is expressed in terms of monomer units.

These surface coverages can be used to estimate a physical film thickness, as follows. The molar volume of monomer units, $V_{\mathrm{m}} / \mathrm{cm}^{3} \mathrm{~mol}^{-1}$ (i.e. the reciprocal of the volume concentration of monomer units), can be estimated as the quotient of monomer molar mass and density, $\mathrm{RMM} / \rho$. The approximation here is that the monomer units in the polymer pack essentially the same as in pure monomer. For a compact, solvent-free ("dry") polymer, the film thickness $h^{*}=V_{\mathrm{m}} I$. The RMM values of PyFMOC and $\mathrm{PyNH}_{2}$ are 344 and $122 \mathrm{~g} \mathrm{~mol}^{-1}$, respectively. Their respective densities are $c a .1 .2$ and $1.0 \mathrm{~g} \mathrm{~cm}^{-3}$. Combining these with the values of $\Gamma_{\text {PिyFMOC }}$ and $\Gamma_{\mathrm{PPyNH} 2}$ from the previous paragraph, we estimate "dry" (i.e. collapsed, solventfree) film thicknesses of $c a$. $600 \AA$ and $240 \AA$ for the PPyFMOC and $\mathrm{PPyNH}_{2}$ films, respectively. Looking ahead to the NR part of the study, solvation might be expected to increase these values by $50-100 \%$, since typical solvent volume fractions for electroactive polymer films are in the range $0.3<\phi_{\mathrm{S}}<0.5 .{ }^{51-54}$ This overall scenario is appropriate both for a NR experiment (to explore structure) and filling of the interridge "trenches" in a typical fingerprint deposit (to accomplish practical visualization). As a final observation, the different thicknesses of these two PPyFMOC and $\mathrm{PPyNH}_{2}$ films containing essentially the same number of monomer units (irrespective of whether one compares two dry films or two solvated films) shows the potential for generation of free volume by FMOC elution from a film.

\section{Observation of changes in surface composition using spectroscopic measurements}

Before addressing the more sophisticated issue of spatial distribution of active components within the film, it is necessary to demonstrate that the strategy of Fig. 3 does indeed result in fluorophore immobilization. In principle, one might consider accomplishing this using either a fluorescence measurement or some other 
spectroscopic probe. Superficially, a fluorescence measurement has the attraction of also providing a more direct functional appraisal. However, while this could demonstrate the presence of the fluorophore, it might not do so if quenching were an issue; in either instance, it would not provide evidence of its immobilization. The concern here is that, unless covalent attachment to the polymer is achieved, facile entry of the fluorophore could just as easily be followed by its elution upon further exposure to electrolyte, thereby jeopardizing the entire surface synthetic strategy. Consequently, we sought a spectroscopic probe able to provide direct evidence of fluorophore-polymer binding. Qualitatively, this can be accomplished using vibrational spectroscopy, with particular focus on the presence (or absence) of carbonyl bands associated with the amide functionality, which will be present in an PPyFMOC film (at the start) and a successfully functionalized PPy-Dylight film (at the end), but should be absent in $\mathrm{PPyNH}_{2}$ (following hydrolysis of the PPyFMOC film).

Representative data are shown in Fig. 6. Trace (a), representing a PPyFMOC film, has a strong absorption band at $1660 \mathrm{~cm}^{-1}$. Trace (b), for the hydrolysed film, has no significant amide band, demonstrating removal of the FMOC functionality. Trace (c), for the hydrolysed film after exposure to Dylight solution, shows a strong band at $1630 \mathrm{~cm}^{-1}$, showing the formation of an amide. Significantly, the last of these observations demonstrates not only the permeation of fluorophore into the film but also its chemical immobilization. Overall, these data show elution of FMOC and binding of fluorophore, but give no insight into the spatial distribution of the fluorophore or the factor(s) limiting its final population. We now address these issues using NR.

\section{Determination of vertical spatial structure using neutron reflectivity}

The second stage of the strategy in Fig. 3 is the creation of free volume in the PPyFMOC film by the hydrolysis and elution of the FMOC moieties. Importantly,

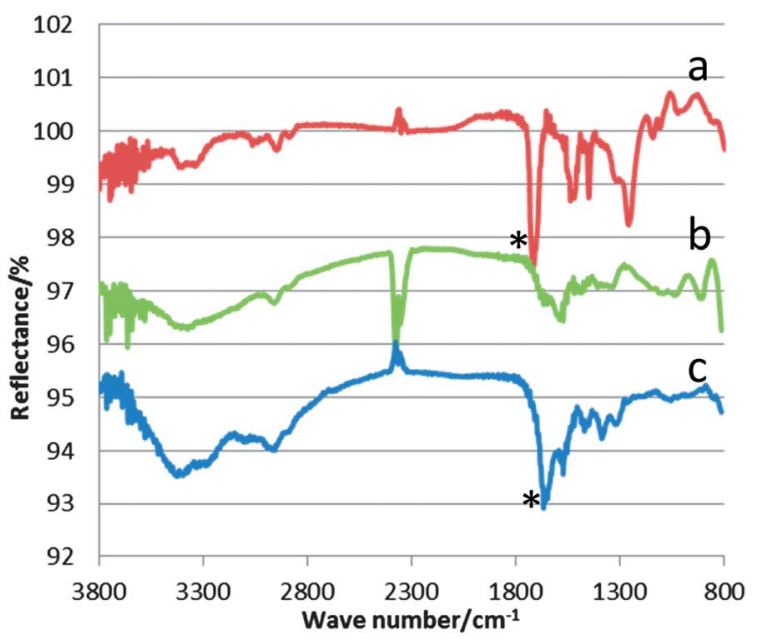

Fig. 6 Reflectance infra-red spectra for a PPyFMOC film subject to the reaction sequence of Fig. 3. Trace (a): PPyFMOC film, as deposited; trace (b): PPyFMOC film after hydrolysis, notionally a $\mathrm{PPyNH}_{2}$ film; trace (c): film of panel (b) after reaction with Dylight solution. Traces are arbitrarily offset vertically for visual clarity. Reaction conditions as in main text. The asterisks on traces (a) and (c) indicate the amide peaks referred to in the main text. 
we require this free volume to be created throughout the film. Fig. 7 shows neutron reflectivity data acquired at different times during the hydrolysis process, conducted in a deuterated solvent medium to optimise the contrast. The data shown in Fig. 7 in fact represent the second - and, in reaction terms, productive phase of the experiment. Since there was no means a priori to predict the timescale of the hydrolysis-elution process, the film was initially exposed to a low $(0.01$ $\mathrm{mM}$ ) concentration of piperidine, with the aim of slowing the reaction to a measurable rate. This approach turned out to be more than successful, in that the rate of hydrolysis was immeasurably slow. However, this had the advantage of providing a film solvation profile at a true " $t=0$ "; the outcome of this is cited below in the discussion of Fig. 7c. The hydrolysis solution was then exchanged to higher piperidine concentration $(10 \mathrm{mM})$ and the data shown in Fig. 7a acquired.
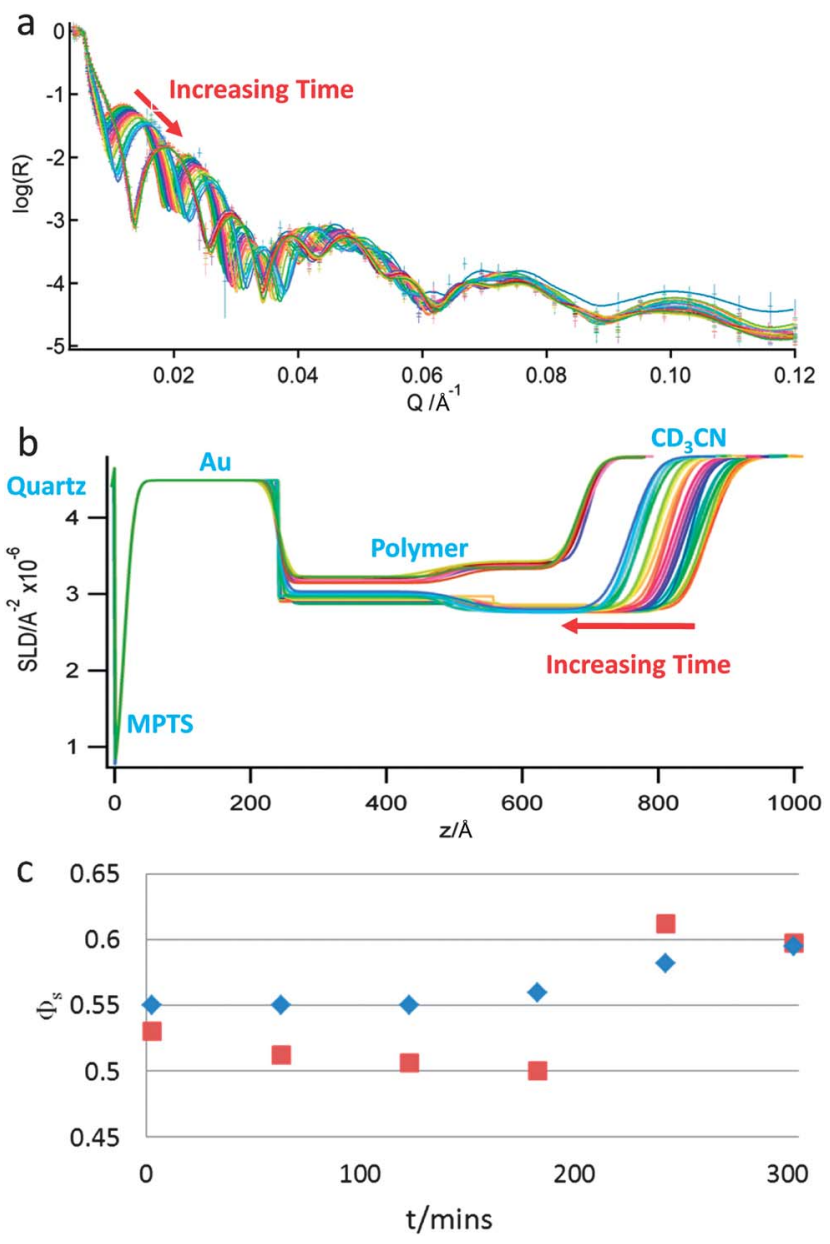

Fig. 7 Time-resolved NR experiment for PPyFMOC hydrolysis to give PPy-voids. Panel (a): $R(Q)$ profiles as a function of time (indicated by arrows); panel (b) model fitted scattering length density profiles as a function of time (from the data of panel (a)); panel (c): solvent volume fraction in the film at selected time intervals (see main text for comment on absolute values) during the hydrolysis. ( $\square$ : inner polymer layer; $\checkmark$ : outer polymer layer). 
The time taken for cell mounting and alignment and for initiation of data acquisition was on the order of $30 \mathrm{~min}$. Hence the time axis in Fig. $7 \mathrm{c}$ has an offset of this order; since there is no attempt to extract kinetic or diffusional parameters, this is not critical.

Broadly speaking, the $R(Q)$ profiles of Fig. 7a comprise three regions. At low $Q$ (here, $Q<0.0055 \AA^{-1}$ ) there is total external reflection $(R=1)$; this is a consequence of the substrate material the neutrons are transmitted through (quartz) having a lower scattering length density than the material they are reflecting from (the deuterated solvent). Beyond this, there is a series of fringes whose periodicity is dictated by the thickness of the films present (Au electrode and polymer) and whose amplitude is dictated by the scattering length density contrast and sharpness of the interfaces between layers. At high $Q$ (here, $Q>0.06 \AA^{-1}$ ), the fringes seen are attributable to the Au electrode. Although these are not central to the aims of the experiment per se, their quantitation does assist fitting of the full $R(Q)$ profile from which we extract the polymer film data. At intermediate $Q$, the Au-derived fringes are seen, but superimposed on these are fringes that result from the polymer film. The latter do not persist to high $Q$ as a consequence of the diffuse polymer/solution interface.

Independent of any model, we can make four deductions from the data. Trivially, the presence of a critical edge shows that the film scattering length density is below that of the solution. The position of the critical edge, $Q^{*}=(16 \pi \Delta N b)^{1 / 2}$, where $\Delta N b$ corresponds to the difference in scattering length densities between the two bulk phases in the sample. In this instance, the relevant bulk phases are the quartz block supporting the electrode and the bathing solution to which the film is exposed; the scattering length densities are known for these materials, giving $\Delta \mathrm{Nb}$ $=0.62 \times 10^{-6} \AA^{-2}$. Inserting this into the expression above, we estimate $Q^{*} \sim$ $0.0056 \AA^{-1}$, consistent with the experimental data of Fig. 7. Secondly, the time invariance of the fringes at high $Q$ allows these to be assigned to the Au electrode, the composition and thickness of which (necessarily) do not vary with time. Thirdly, an Au electrode thickness of $210 \AA$ can be estimated from the periodicity of the fringes, $\Delta Q=0.03 \AA^{-1}$, via the equation $\Delta Q=2 \pi / d$ (where $d=$ film thickness); this is entirely consistent with the nominal thickness of $200 \AA$ from the sputtering process. Fourthly, the higher frequency fringes present only in the intermediate $Q$ region can be seen progressively to stretch out with time. Since the momentum transfer, $Q$, is in reciprocal space, this stretching corresponds to some element of contraction of film thickness with hydrolysis of the FMOC groups. The quantitative question that follows is whether this contraction results in loss of some or all of the "void" volume generated by the FMOC departure.

To address this last question, detailed fitting of the data is required. Full details of this standard procedure are given in the Experimental section and elsewhere, ${ }^{47-49}$ but the essential points are as follows. The scattering lengths of all the components present (quartz, Au, PPyFMOC, the departing FMOC, the remaining PPy-amine and $\mathrm{CD}_{3} \mathrm{CN}$ solvent) are all known. The Au thickness is known (from a combination of fabrication protocol, bare electrode observations and the high Q data of Fig. 7). The unknowns are therefore the internal composition of the film (predominantly, solvation level), film thickness and the roughness of the polymer/solution interface.

The outcomes of the fitting process are shown in Fig. 7b and 7c. In the first of these, the scattering length density of the system, from the quartz block supporting the electrode through to the bulk solution, is shown as a function of 
distance, $z$ (perpendicular to the interface). The values for quartz, Au and solvent represent bulk values for the pure components. The sharp dip between the quartz support and the Au electrode represents the MPTS bonding layer used to ensure good $\mathrm{Au}$ adhesion. For the purposes of this work, the region of interest is from the $\mathrm{Au}$ electrode outwards. The interface between the $\mathrm{Au}$ and the film is relatively sharp; a small amount of diffuseness (typically $10 \AA$ ) is required to account for the finite roughness of the Au surface. Interestingly, the polymer film is not compositionally homogeneous at any point in the process: there is a relatively diffuse outer region (a transition from "bulk" film to bulk solution over $40 \AA$ ) and the interior of the film comprises compositionally distinct inner and outer regions. A single polymer layer with high interfacial roughness was insufficient to model the film. The key outcome is a substantial shrinkage of the film as hydrolysis proceeds. For the example shown, the dry film thickness (measured in air) was $561 \AA$, and the solvated film thickness (measured upon exposure to solvent, prior to hydrolysis) was $639 \AA$, and the latter shrunk to $443 \AA$ following completion of FMOC hydrolysis and elution.

The significance of this in solvation terms is shown in the solvent volume fraction data of Fig. 7c. While the fitting unquestionably demands that some film inhomogeneity be recognized (see Fig. 7b), the difference in scattering length density (and thence solvation) between the inner and outer regions of the film is relatively small. We therefore look at the average picture. The film solvent volume fraction at the outset, as solvated PPyFMOC exposed to a low piperidine concentration (as explained above) is 0.41 . By the end of the hydrolysis process, this increases to 0.60 , but it subsequently falls to 0.52 . The latter decrease is the result of polymer relaxation, which occurs on a longer timescale than the FMOC hydrolysis and elution.

It is of course not possible to make the analogous measurements in hydrogenous solvent $\left(\mathrm{CH}_{3} \mathrm{CN}\right)$ for the same film, since the hydrolysis reaction is a onetime process. However, such measurements were made on a nominally identical film and the outcome, in summary form for the analogue of Fig. 7c, was an initial solvent volume fraction of 0.35 , rising to 0.58 immediately after hydrolysis and subsequently relaxing back to 0.44 . The common conclusion from these experiments is that FMOC removal increases the film solvent volume fraction by $\Delta \phi_{\mathrm{S}} \sim$ 0.2 in the short term, but this increase is subsequently diminished to $\Delta \phi_{\mathrm{S}} \sim 0.1$. While this may seem modest, we note that relatively small changes in solvent content can have profound effects on polymer chain mobility, for example as manifested in viscoelastic properties,${ }^{54,55}$ which would facilitate permeation of fluorophore reactant. In an absolute sense, a replaceable solvent volume fraction $\phi_{\mathrm{S}}>0.4$ is more than adequate to give a high (and thus visible) fluorophore population.

This leads to consideration of the final step in the scheme of Fig. 3, fluorophore functionalization of the $\mathrm{PPy}-\mathrm{NH}_{2}$ film. What reaction did occur - and the significant changes in $R(Q)$ profiles do unequivocally demonstrate change, quantified below - took place within the first $15 \mathrm{~min}$ of exposure to fluorophore solution. We attribute this to the more fluid-like environment of the film following hydrolysis (see above). From a mechanistic perspective, this removed the opportunity to follow the kinetics of the process (largely due to instrumental issues such as sample alignment prior to measurement), but from a practical perspective in future application it is obviously beneficial. 
Consequently, on a separate (but nominally similar) film to that of Fig. 7, a separate set of measurements were made, as follows. First, $R(Q)$ data were acquired for a PPyFMOC film in the dry state (solvent-free) and exposed to $h_{3}$ - and $d_{3}$-acetonitrile, prior to hydrolysis. Second, the film was hydrolysed (with no attempt to monitor the time-dependence of this process) and $R(Q)$ data acquired for the resultant $\mathrm{PPyNH}_{2}$ film in the three environments (air, $h_{3^{-}}$and $d_{3}$-acetonitrile). Finally, the film was Dylight functionalised and $R(Q)$ data acquired in the same three environments. This last part of the experiment is complementary to the hydrolysis step, in that the aim is entry of a large reactant to consume free volume, rather than elution of a large leaving group to generate free volume. As compensation for sacrifice of any kinetic information, this suite of measurements provided data in different solvent contrasts for the same film, which (through corefinement) gives greater certainty in fitting. The resulting $R(Q)$ profiles are shown in Fig. 8a, grouped according to the film environment; the general form of the profiles is analogous to those of Fig. 7.

The key parameters of interest are film thickness and solvent content at each stage. To extract these, we need to consider the contributions of the polypyrrole spine, fluorophore and solvent components to the scattering length density. For the polypyrrole and solvent components, the scattering length and physical density are known. For the fluorophore, whose structure is commercially protected, this is not so straightforward, but acceptable approximations are possible,
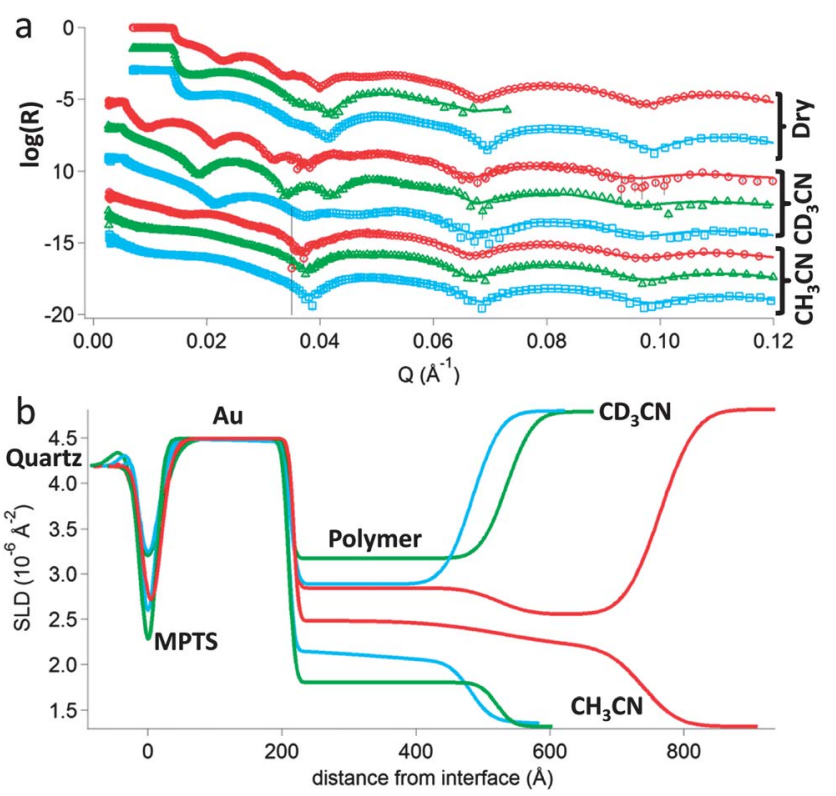

Fig. 8 Panel (a): $R(Q)$ data for a PPyFMOC film prior to hydrolysis $(O)$, the $\mathrm{PPyNH}_{2}$ film resulting from hydrolysis $(\triangle)$ and the PPy-Dylight film following exposure to the fluorophore ( $\square$ ). Points represent data; lines represent fits (see panel (b)). For visual comparison purposes, $R(Q)$ profiles are group according to the ambient medium (see annotations). Data are progressively offset downwards for presentational purposes; for the dry and $d_{3}$-acetonitrile exposed films, a critical edge is seen, below which $R=1$. Panel (b): model fitted scattering length density profiles for the film exposed to $h_{3^{-}}$ acetonitrile and $d_{3}$-acetonitrile at each of the three stages of the process: nominally PPyFMOC (red traces), $\mathrm{PPyNH}_{2}$ (green traces) and PPy-Dylight (blue traces). 
as follows. The molar mass is on the order of 1000 Daltons and there are four sulfonate groups (to provide adequate solubility) on an essentially aromatic hydrocarbon skeleton. We thus have an entity that comprises $c a .320$ Daltons of " $\mathrm{SO}_{3}{ }^{-}$" and $c a$. 680 Daltons of " $\mathrm{CH}$ "; we make the plausible approximation that the overall density is unity. With these physically reasonable approximations, combining the data sets for the $h_{3}$-acetonitrile and $d_{3}$-acetonitrile environments, we estimate that there is one fluorophore entity for every $c a$. five pyrrole monomer units. Physically, this is plausible, given the geometrical constraints of the cartoon representation of Fig. 3 and practically this is expected to be useful.

The model scattering length density profiles best fitting the data are shown in Fig. $8 \mathrm{~b}$ and the $R(Q)$ fits (lines) are shown alongside the data (points) in Fig. 8a; the agreement is good across the accessible $Q$ range. We particularly highlight four characteristics. First, the outer interfaces are diffuse. In the $R(Q)$ profiles, this accounts for the damping of the film-based fringes. In terms of reactivity, it undoubtedly contributes to the faster permeation of the fluorophore molecules. Second, once one progresses to the interior of the film, its scattering length density, and thus composition, shows at most only modest dependence on depth (actually, none for $\mathrm{PPyNH}_{2}$ ). This indicates that diffusion of reactant into the film is not a limiting factor; if it were, then there would be a clear gradient of composition representing fluorophore penetration. Third, despite the entry of fluorophore into the polymer, the films shrink slightly during the process (compare the traces for $\mathrm{PPyNH}_{2}$ and PPy-Dylight). This indicates that the volume of solvent expelled exceeds the volume of fluorophore entering, suggesting that transport processes are not so slow that mobile species (here, solvent) are trapped within the film. Finally, while the outer interface is slightly sharper for the dry film (profile not shown), the overall thickness is not much less than for the solventexposed film. Since (see Table 1) there is still appreciable solvent in the immersed films, this suggests that the film does not collapse upon emersion.

The characteristics of the model profiles are summarized in Table 1. Considering first the thickness data, the dramatic collapse of the PPyFMOC film upon hydrolysis (by ca. 40\%) is accompanied by an increase in solvent content of the resulting $\mathrm{PPyNH}_{2}$ film. This apparently counter-intuitive result is a consequence of the size of the FMOC group; recall the earlier estimations of film thickness accompanying the coulometry, when it was noted that the FMOC group constitutes $c a .60 \%$ of the film volume. Turning to the solvent volume fraction, the values for PPyFMOC $\left(\phi_{\mathrm{S}}=0.36\right)$ and $\mathrm{PPyNH}_{2}\left(\phi_{\mathrm{S}}=0.47\right)$ are satisfyingly consistent

Table 1 Summary of film thickness and solvent content values at each stage of assembly of the surface architecture represented schematically in Fig. 3. $\mathrm{PPyNH}_{2}$ films could be modelled as a single layer (i.e. were internally homogeneous), so "inner" and "outer" regions are merged

\begin{tabular}{|c|c|c|c|c|c|c|}
\hline \multirow{2}{*}{$\begin{array}{l}\text { Environment } \\
\text { Parameter }\end{array}$} & \multicolumn{2}{|c|}{ Air (“dry”) } & \multicolumn{4}{|c|}{ Exposed to acetonitrile } \\
\hline & \multicolumn{2}{|c|}{ Thickness/Å } & \multicolumn{2}{|c|}{ Thickness/Å } & \multicolumn{2}{|c|}{$\begin{array}{l}\text { Solvent volume } \\
\text { fraction } / \varphi_{\mathrm{S}}\end{array}$} \\
\hline Film region & Inner & Outer & Inner & Outer & Inner & Outer \\
\hline PPyFMOC & 173 & 303 & $312( \pm 18)$ & $223( \pm 4)$ & 0.34 & 0.38 \\
\hline $\mathrm{PPyNH}_{2}$ & \multicolumn{2}{|c|}{257} & \multicolumn{2}{|c|}{$318( \pm 8)$} & \multicolumn{2}{|c|}{0.47} \\
\hline PPy-Dylight & 104 & 164 & $188( \pm 3)$ & $84( \pm 3)$ & 0.40 & 0.62 \\
\hline
\end{tabular}


with those of $\phi_{\mathrm{S}}=0.38$ and $\phi_{\mathrm{S}}=0.48$ at the same points in the surface chemistry of Fig. 3 determined by averaging the outcomes of the two kinetic experiments of Fig. 7 and its $h_{3}$-acetonitrile counterpart.

\section{Functional viability of the fluorophore-modified film}

This report focuses on construction of the interfacial architecture and, via the NR measurements, on establishing spatial control of fluorophore immobilization within the polymer matrix; essentially, this represents the compositional and structural aspects of the strategy. In a subsequent phase of the work, the focus will shift to determination of the polymer chromophore and immobilized fluorophore properties; these represent the functional aspects of the strategy. The latter will involve a substantive programme of measurements, notably as functions of excitation and observational wavelengths and of polymer charge state (i.e. doping level, manipulated via applied potential). Nonetheless, in advance of such a future report, there is merit in a forward look to establish at a qualitative level fluorophore activity in the polymer film context. The technical issue here is whether (or not) proximity of the fluorophore sites to the underlying electrode results in fluorescence quenching.

Fig. 9 shows two images of PPy-based films representing two stages simplistically, in the absence and presence of fluorophore, respectively - of the assembly process shown in Fig. 3. This preliminary observation does not attempt to address the spatial issue of imaging a full fingerprint, but focuses solely on the viability of fluorophore emission when in the film environment. The left hand image shows a section of a $\mathrm{PPyNH}_{2}$ film deposited on an $\mathrm{Au}$ substrate (using the procedure of Fig. 5, terminating at the cathodic end of a potential cycle to establish the undoped redox state), removed from solution and viewed ex situ (dry) under illumination by light of wavelength $640 \mathrm{~nm}$. This control observation shows a few brighter areas, but nothing systematic or substantive. The right hand image shows

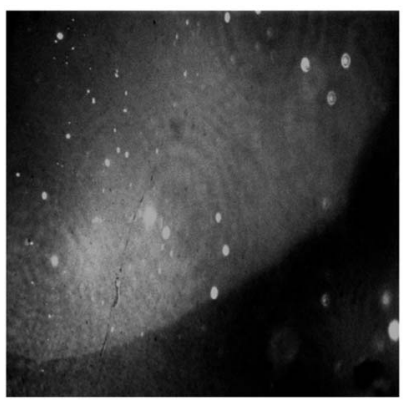

Panel $a$

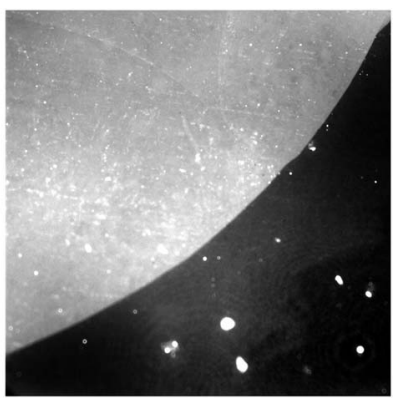

Panel $b$

Fig. 9 Panel (a): PPy film on Au (deposition procedure as in main text); this represents the control experiment, in the absence of fluorophore. Panel (b): $\mathrm{PPyNH}_{2}$ film prepared by hydrolysis of a PPyFMOC film (deposition and subsequent treatment as in main text) after partial exposure to Dylight 649 NHS ester by contact with a droplet of fluorophore solution, and subsequent removal of excess fluorophore by rinsing. The top left part of the viewed region includes part of the droplet-exposed area. In both cases, the films were in the reduced (undoped) state and were viewed ex situ under illumination by light of wavelength $640 \mathrm{~nm}$. In panel (b), the intensity was attenuated by a factor of 4 . 
a partially Dylight 649 ester functionalised film, prepared as follows. A PPyFMOC film was deposited on $\mathrm{Au}$ and hydrolysed (as discussed earlier with reference to Fig. 7), then a droplet of Dylight 649 NHS ester solution placed on one part of the surface, followed by rinsing with pure water (to remove unbound and surface/ exterior fluorophore) and air drying (at room temperature for $c a .15 \mathrm{~min}$ ). The resultant film was viewed ex situ under illumination with light of wavelength $640 \mathrm{~nm}$. The top left part of the viewed region in panel (b) of Fig. 9 includes part of the droplet-exposed area. The sharply defined region of enhanced brightness is consistent with strong fluorophore emission; note that the intensity was attenuated (see legend) so the distinction between the images in panels (a) and (b) is significant. Although this is not a quantitative measure of the fluorescence efficiency and does not totally exclude quenching - perhaps from fluorophores sites closer to the electrode (see Fig. 8 for evidence of deep penetration of fluorophore) - it is clear that at least some fluorophore sites are sufficiently distant from the electrode that they are not vulnerable to quenching. Pragmatically, the practical viability of the interfacial (electro)chemical strategy is established.

\section{Conclusions}

A combination of spectroscopic, electrochemical and neutron-based techniques provides the capability to follow and quantify deposition and subsequent functionalization of electroactive polymer films relevant to latent fingerprint visualization. This approach has been used to explore the (electro)chemistry of pyrrole-FMOC electropolymerization and deposition, followed by hydrolysis of the FMOC leaving group, then permeation and bonding of the fluorophore Dylight 649 NHS ester. By revealing the presence, removal and reintroduction of amide functionalities, FTIR spectroscopy demonstrates qualitative success of this postdeposition functionalization strategy. Electrochemistry provides control over (and coulometric assay of) the surface population of polymer, the electrochromic matrix into which the fluorophore is introduced. Neutron reflectivity provides insight into the vertical spatial distribution of the permeating fluorophore and the changes in film population of the solvent that must leave to create space for it. Together, these techniques provide insights into film composition, structure and (in the cases of the electrochemical and neutron data) dynamics; simplistically, they address the tersely expressed questions "what, how much and where?"

We have demonstrated that the portfolio of materials suitable for electrochromic enhancement of latent fingerprints can be extended from the previously used aniline and thiophene (PEDOT) families to include pyrrole-based materials. Specifically, this was accomplished for the parent polypyrrole, $N$-propylamine functionalised pyrrole and FMOC functionalised pyrrole. In future, in addition to the substitution chemistry explored here, this will provide a wider colour palette with which to optimise latent fingerprint visual contrast against the substrate.

In the post-deposition functionalization of PPyFMOC, the hydrolysis process (leading to FMOC removal) is relatively slow (ca. $3 \mathrm{~h}$ under the conditions employed), which allowed the progress of the reaction to be monitored by neutron reflectivity. The subsequent entry of fluorophore (Dylight 649 NHS ester) is much more rapid - too rapid to follow readily using neutron reflectivity - which is attributed to the higher solvent volume fraction and thence greater fluidity of the film. During this sequence of events, the solvent volume fraction rises by $c a$. 
0.2 immediately upon hydrolysis and FMOC elution, but then falls by ca. 0.1 , presumably as a consequence of polymer relaxation in the now highly plasticised film. In absolute terms, the solvent volume fractions are $0.37( \pm 0.03)$ for PPyFMOC prior to hydrolysis, $0.59( \pm 0.01)$ immediately after hydrolysis and $0.47( \pm 0.04)$ after relaxation (where these data originate from both kinetic and non-kinetic experiments). After fluorophore entry, the film contracts slightly.

Future prospects for a combined absorption/fluorescence strategy in latent fingerprint enhancement appear promising. The next step is implementation of the strategy described here to fingerprinted surfaces. Having established control of reactivity and composition in the vertical direction at distance scales from 10-100 $\mathrm{nm}$, this fine control can now be applied to the lateral direction. Since the fingerprint feature sizes are at the scale of $>10 \mu \mathrm{m}$, the prospect of high resolution, high fidelity fingerprint images is excellent.

\section{Acknowledgements}

We thank the Institut Laue Langevin and ISIS Facility of the Rutherford Appleton Laboratory for provision of neutron beam time. R. M. B. thanks the University of Leicester for financial support. We thank Ines Carro-Muino (of Thermo Fisher Scientific) for helpful conversations relating to fluorophores and Zeta Instruments for helpful discussions regarding sample analysis using 3D optical profiling.

\section{References}

1 Advances in Fingerprint Technology (2nd edn), ed. H. C. Lee and R. E. Gaensslen, CRC Press, Boca Raton, 2001.

2 R. S. Ramotowski, in Advances in Fingerprint Technology (2nd edn), ed. H. C. Lee and R. E. Gaensslen, CRC Press, Boca Raton, 2001, p. 63.

3 R. D. Olsen and H. D. Lee, Advances in Fingerprint Technology (2nd edn), ed. H. C. Lee and R. E. Gaensslen, CRC Press, Boca Raton, 2001, p. 41.

4 P. Voss-De Haan, Contemp. Phys., 2006, 47, 209.

5 J. Berry and D. A. Stoney, in Advances in Fingerprint Technology (2nd edn), ed. H. C. Lee and R. E. Gaensslen, CRC Press, Boca Raton, 2001, p.1.

6 J. G. Barnes, in The Fingerprint Source Book, ed. A. McRoberts, National Institute of Justice, Washington, 2011, ch. 1. Available from http://www.nij.gov/pubs-sum/225320.htm.

7 W. J. Herschel, Nature, 1880, 23, 76.

8 K. Barnett, in Crime Scene to Court: the Essentials of Forensic Science (1st edn), ed. P. White, Royal Society of Chemistry, Cambridge, UK, 1998, p. 98.

9 F. Galton, Finger Prints, Macmillan, London, 1892.

10 H. Faulds, Nature, 1880, 22, 605.

11 M. Kucken, Forensic Sci. Int., 2007, 171, 85.

12 I. W. Evett and R. L. Williams, J. Forensic Ident., 1996, 46, 49.

13 Advances in Fingerprint Technology (3rd edn), ed. R. S. Ramotowski, CRC Press, Boca Raton, 2013.

14 S. Bell, Annu. Rev. Anal. Chem., 2009, 2, 297.

15 T. A. Brettell, J. M. Butler and J. R. Almirall, Anal. Chem., 2011, 83, 4539.

16 N. Akiba, N. Saitoh and K. Kuroki, J. Forensic Sci., 2007, 55, 180.

17 M. J. Choi, T. Smoother, A. A. Martin, A. M. McDonagh, P. J. Maynard, C. Lennard and C. Roux, Forensic Sci. Int., 2007, 173, 154.

18 J. Almog, G. Levinton-Shamuilov, Y. Cohen and M. Azoury, J. Forensic Sci., 2007, 52, 1057.

19 J. D. James, C. A. Pounds and B. Wilshire, J. Forensic Sci., 1993, 38, 391.

20 L. K. Seah, U. S. Dinish, W. F. Phang, Z. X. Chao and V. M. Murukeshan, Forensic Sci. Int., 2005, 152, 249.

21 V. Bowman, Manual of Fingerprint Development Techniques (2nd edn), Police Scientific Development Branch, Home Office, Sandridge, UK, 2004.

22 H. L. Bandey and T. Kent, Superglue Treatment of Crime Scenes 30/03, Police Scientific Development Branch, Home Office, Sandridge, UK, 2003. 
23 A. A. Cantu, D. A. Leben, R. Ramotowski, J. Kopera and J. R. Simms, J. Forensic Sci., 1998, 43, 294.

24 C. Kauffman and K. Smith, J. Forensic Ident, 2001, 51, 9.

25 Y. Migron and D. Mandler, J. Forensic Sci, 1997, 42, 986.

26 G. S. Sodhi and J. Kaur, Forensic Sci. Int., 2001, 120, 172.

27 A. R. W. Jackson and J. M. Jackson, Forensic Science, Pearson Prentice House, Harlow, UK, 2004.

28 A. A. Cantu, in Optics and Photonics for Counterterrorism and Crime Fighting III, SPIE-Int. Soc. Opt. Eng., ed. C. Lewis, 2007, vol. 6741, p. D7410.

29 P. Czekanski, M. Fasola and J. Allison, J. Forensic Sci., 2006, 51, 1323.

30 M. Colella, A. Parkinson, T. Evans, C. Lennard and C. Roux, J. Forensic Sci., 2009, 54, 583.

31 P. F. Kelly, R. S. P. King and R. J. Mortimer, Chem. Commun., 2008, 6111.

32 K. K. Bouldin, E. R. Menzel, M. Takatsu and R. H. Murdock, J. Forensic Sci., 2000, 45, 1239.

33 R. Leggett, E. E. Lee-Smith, S. M. Jickells and D. A. Russell, Angew. Chem., Int. Ed., 2007, 46, 4100.

34 A. L. Beresford and A. R. Hillman, Anal. Chem., 2010, 82, 483.

35 R. M. Brown and A. R. Hillman, Phys. Chem. Chem. Phys., 2012, 14, 8653.

36 C. Bersellini, L. Garofano, M. Giannetto, F. Lusardi and G. Mori, J. Forensic Sci, 2001, 46, 871.

37 A. L. Beresford, R. M. Brown, A. R. Hillman and J. W. Bond, J. Forensic Sci., 2012, 57, 93.

38 F. Cortes-Salazar, J. M. Busnel, F. Li and H. H. Girault, J. Electroanal. Chem., 2009, 635, 69.

39 M. Zhang and H. H. Girault, Analyst, 2009, 134, 25.

40 F. Cortes-Salazar, M. Q. Zhang, A. Becue, J. M. Busnel, M. Prudent, C. Champod and H. H. Girault, Chimia, 2009, 63, 580.

41 M. Q. Zhang and H. H. Girault, Electrochem. Commun., 2007, 9, 1778.

42 G. Qin, M. Zhang, Y. Zhang, Y. Shu, S. Liu, W. Wu and X. Zhang, J. Electroanal. Chem, 2013, 693, 12.

43 A. Glidle, A. R. Hillman, K. S. Ryder, E. L. Smith, J. M. Cooper, N. Gadegaard, J. R. P. Webster, R. M. Dalgliesh and R. Cubitt, Langmuir, 2009, 25, 4093.

44 A. Glidle, A. R. Hillman, K. S. Ryder, E. L. Smith, J. M. Cooper, R. M. Dalgliesh, R. Cubitt and T. Geue, Electrochim. Acta, 2009, 55, 439.

45 R. A. Campbell, H. P. Wacklin, I. Sutton, R. Cubitt and G. Fragneto, Eur. Phys. J. Plus, 2011, 126, 107, DOI: 10.1140/epjp/i2011-11107-8.

46 J. R. P. Webster, S. Langridge, R. M. Dalgliesh and T. R. Charlton, Eur. Phys. J. Plus, 2011, 126, 112, DOI: 10.1140/epjp/i2011-11112-y.

47 J. Penfold, R. M. Richardson, A. Zarbakhsh, J. R. P. Webster, D. G. Bucknall, A. R. Rennie, R. A. L. Jones, T. Cosgrove, R. K. Thomas, J. S. Higgins, P. D. I. Fletcher, E. Dickinson, S. J. Roser, I. A. McLure, A. R. Hillman, R. W. Richards, E. J. Staples, A. N. Burgess, E. A. Simister and J. W. White, J. Chem. Soc., Faraday Trans., 1997, 93, 3899.

48 A. Glidle, J. Cooper, A. R. Hillman, L. Bailey, A. Jackson and J. R. P. Webster, Langmuir, 2003, 19, 7746.

49 A. Nelson, J. Appl. Crystallogr., 2006, 39, 273.

50 S. H. Cho, K. T. Song and J. Y. Lee, in Handbook of Conducting Polymers (3rd edn), ed. T. A. Skotheim and J. R. Reynolds, CRC Press, Boca Raton, 2007, ch. 8, pp. 8-3.

51 I. Efimov and A. R. Hillman, Anal. Chem., 2006, 78, 3616.

52 A. Glidle, A. R. Hillman, K. S. Ryder, E. L. Smith, J. M. Cooper, N. Gadegaard, J. R. P. Webster, R. M. Dalgliesh and R. Cubitt, Langmuir, 2009, 25, 4093.

53 A. Glidle, A. R. Hillman, K. S. Ryder, E. L. Smith, J. M. Cooper, R. M. Dalgliesh, R. Cubitt and T. Geue, Electrochim. Acta, 2009, 55, 439.

54 A. R. Hillman, M. A. Mohamoud and I. Efimov, Anal. Chem., 2011, 83, 5696.

55 A. R. Hillman, I. Efimov and K. S. Ryder, J. Am. Chem. Soc., 2005, 127, 16611. 